\title{
Complicaciones de fracturas supracondíleas humerales en niños
}

\author{
Complications of supracondylar humeral fractures in children \\ Canales-Zamora OA, * Mora-Ríos FG, ${ }^{\ddagger}$ Mejía-Rohenes LC, ${ }^{\S}$ \\ Anaya-Morales A, " González-Gijón OR, * López-Hernández JR" \\ Hospital Regional «General Ignacio Zaragoza», ISSSTE, México, Ciudad de México.
}

RESUMEN. Introducción: Las fracturas supracondíleas representan la causa más frecuente de las lesiones del codo pediátrico (64\% de la población en niños menores de ocho años). Estas fracturas pueden presentar complicaciones como: A) complicaciones previas al tratamiento: 1) neurológicas, 2) vasculares y 3) síndrome compartimental. B) complicaciones posteriores al tratamiento: 1) precoces en los primeros días tras el tratamiento: pérdida de reducción, complicaciones neurológicas, vasculares, síndrome compartimental, infección de las agujas de Kirschner, 2) complicaciones tardías en el tratamiento: deformidad angular, pérdida de movilidad, miositis osificante, necrosis avascular de la tróclea, entre otros. Objetivo: Determinar la frecuencia de las complicaciones en fracturas supracondíleas humerales en menores de ocho años. Material y métodos: Se realizó un estudio retrospectivo y observacional de cohorte en pacientes menores de ocho años en el período de Marzo de 2014 a Febrero de 2018. Resultados: Se obtuvieron 277 pacientes con los siguientes porcentajes: 3.97\% presentó lesiones de cúbito varo; $1.44 \%$, lesiones neurológicas previas al tratamiento quirúrgico; $1.44 \%$, complicaciones neurológicas precoces al tratamiento; $0.72 \%$, infecciones de agujas de Kirschner; $0.72 \%$, cúbito valgo y $0.36 \%$ con pérdida de la movilidad. Conclusiones: La complicación más frecuente de las fracturas supracondíleas de húmero en este estudio fue cúbito varo y complicaciones neurológicas previas al tratamiento.
ABSTRACT. Introduction: Supracondylar fractures represent the most frequent cause of pediatric elbow injuries, at $64 \%$, in children under eight years old. These fractures can present complications such as: A) Complications prior to treatment: 1) neurological, 2) vascular and 3) compartment syndrome. B) Complications after treatment: 1) early, in the first days after treatment: loss of reduction, neurological, vascular, compartment syndrome, infection of Kirschner wires. 2) Late complications in treatment: Angular deformity, loss of mobility, ossifying myositis, avascular necrosis of the trochlea, others. Objective: To determine the frequency of complications in humeral supracondylar fractures in less than eight years. Material and methods: A retrospective, observational cohort study was conducted in patients under 8 years of age during the period of March 2014 to February 2018. Results: 277 patients were obtained with the following percentages: cubitus varus $3.97 \%$, neurological lesions prior to surgical treatment $1.44 \%$, early neurological complications to treatment $1.44 \%$, infections of Kirschner needles $0.72 \%$, cubitus valgus $0.72 \%$, loss of mobility $0.36 \%$. Conclusions: The most common complication of humerus supracondylar fractures in this study was cubitus varus and neurological complications prior to treatment.

\section{Nivel de evidencia: IV serie de casos}

* Médico residente del Cuarto año de Ortopedia y Traumatología de la Facultad Mexicana de Medicina, Universidad La Salle. Hospital Regional «General Ignacio Zaragoza», ISSSTE, México, Ciudad de México.

‡ Médico adscrito al Módulo Pediátrico. Hospital Regional «General Ignacio Zaragoza», ISSSTE, México, Ciudad de México.

$\S$ Jefe de Servicio Hospital Regional «General Ignacio Zaragoza», ISSSTE, México, Ciudad de México.

" Médico adscrito al Servicio de Ortopedia Hospital Regional «General Ignacio Zaragoza», ISSSTE, México, Ciudad de México.

๑ Médico residente de Cirugía Articular. Centro Médico Nacional 20 de Noviembre, ISSSTE, México, Ciudad de México.

Dirección para correspondencia:

Dr. Félix Gustavo Mora-Ríos

Avenida Zaragoza Núm. 1711, Col. Ejército Constitucionalista, C. P. 09220, Alcaldía Iztapalapa, CDMX.

Teléfono: 57165200, ext. 16725,

E-mail: drmoraortoped@hotmail.com oskarcanales@hotmail.com

https://dx.doi.org/10.35366/95321

doi: $10.35366 / 95321$ 
Palabras clave: Complicaciones, cúbito, varo, valgo, fractura, niños.

\section{Introducción}

El porcentaje de las fracturas del miembro torácico que corresponde al codo es de 5 a 18\%; de ellas, las fracturas supracondíleas representan la causa más frecuente de lesiones del codo pediátrico con $64 \%$ en menores de ocho años. ${ }^{1,2}$ La fractura supracondílea humeral es la solución de continuidad de la metáfisis distal del húmero por encima de la línea fisaria. ${ }^{3}$ Para la clasificación de fracturas supracondíleas humerales, existen varias opciones; en la actualidad, la clasificación de Gartland es la más usada. ${ }^{4} \mathrm{Al}$ respecto, Gartland clasificó las fracturas supracondíleas por su tipo de extensión y en su papel original de la siguiente manera: ${ }^{5}$

A) Tipo I. Fractura no desplazada $(<2 \mathrm{~mm})$. La línea humeral anterior todavía atraviesa el centro del cóndilo humeral. ${ }^{5}$

B) Tipo II. Moderadamente desplazada (> $2 \mathrm{~mm}$ ). La línea humeral anterior pasa anterior al centro del cóndilo hume$\mathrm{ral}^{5}$

C) Tipo III. Completamente desplazada. Este tipo de fractura es más inestable, se extiende a tejidos blandos, daña al periostio y aumenta las lesiones neurovasculares. ${ }^{5}$

De estas fracturas, en cuanto a su manejo, las tipo I pueden tratarse de forma conservadora, esto es, fracturas sin desplazamiento. Sin embargo, no existe un consenso en cuanto al tratamiento de las fracturas con desplazamiento, es decir, las tipo II y III.

La reducción cerrada y la fijación con agujas percutáneas de Kirschner en disposición lateral o cruzada se ha popularizado hasta convertirse en el método más ampliamente aceptado en la actualidad. ${ }^{6}$ Las fracturas pueden presentar complicaciones, las cuales se mencionan a continuación: ${ }^{7}$

\section{A) Complicaciones previas al tratamiento 7}

Complicaciones neurológicas: la incidencia reportada de lesión nerviosa varía ampliamente de 6 a 42\%, a pesar de que los nervios cubital, radial y mediano están en riesgo de lesión. La lesión aislada del nervio interóseo anterior se ha descrito como la lesión nerviosa predominante. Cramer reporta una tasa de incidencia de lesión aislada del nervio interóseo anterior de $5.9 \%$ en las fracturas supracondíleas humerales. ${ }^{8}$

Complicaciones vasculares: la ausencia de pulso radial se informa en 6 a $20 \%$ de todas las fracturas supracondíleas. ${ }^{9,10}$ El origen del trastorno vascular puede ocurrir por compresión, espasmo del vaso, daño de la íntima, trombo y laceración; en la mayoría de las ocasiones, la lesión vascular se debe a espasmo arterial. ${ }^{6}$
Keywords: Complications, cubitus, varus, valgus, fracture, children.

Síndrome compartimental: el síndrome compartimental es raro, pero debe considerarse como una complicación de un traumatismo de alta energía. ${ }^{11}$ El síndrome compartimental puede ocurrir de 0.1 a $0.3 \%$ de todos los casos. ${ }^{5} \mathrm{La}$ lesión vascular y la inflamación primaria pueden llevar al desarrollo del síndrome compartimental en 12 a 24 horas. Si un síndrome compartimental no es tratado a tiempo, la isquemia asociada puede progresar a un infarto y al desarrollo posterior de la contractura isquémica de Volkmann. ${ }^{9}$

\section{B) Complicaciones posteriores al tratamiento 7 \\ 1. Complicaciones precoces durante los primeros días tras el tratamiento}

- Pérdida de reducción: en las fracturas supracondíleas pediátricas, la reducción cerrada y la fijación percutánea es el tratamiento más ampliamente aceptado. Existen dos configuraciones para colocar las agudas de Kirchner: fijación con entrada lateral y entrada media-lateral cruzada. Aunque la fijación medial-lateral cruzada proporciona más estabilidad biomecánica, conlleva un riesgo de lesión iatrogénica del nervio cubital. ${ }^{12} \mathrm{La}$ pérdida de la reducción puede ocurrir entre 3 a 10\% de los casos. ${ }^{13}$

Complicaciones neurológicas: recientemente, ha habido una serie de estudios donde destaca el tratamiento quirúrgico de las lesiones nerviosas iatrogénicas de fracturas supracondíleas de húmero, que normalmente consiste en la reducción cerrada y la colocación percutánea de agujas de Kirschner ${ }^{14}$ En su revisión sistemática, Brauer encontró que la lesión iatrogénica del nervio se produjo en 3.5\% de los pacientes que tenían los clavillos cruzados y en $1.9 \%$ de los pacientes con colocación de clavos laterales. ${ }^{14} \mathrm{Babal}$, en su revisión sistemática, en el metaanálisis encontró que el nervio más comúnmente implicado en neuropraxia iatrogénica fue el cubital (3.2\%), seguido del mediano (1.8\%) y nervio radial $(1.6 \% .)^{14}$

Complicaciones vasculares: a pesar de la tasa de que existe una tasa de complicaciones relativamente bajo, algunas fracturas de húmero supracondíleas requieren procedimientos operatorios de repetición temprana en el período postoperatorio; sin embargo, estos factores de riesgo para repetir los procedimientos son en gran parte desconocidos. ${ }^{1}$ La lesión vascular puede ocurrir entre 0 y $3 \%$ de los casos. ${ }^{13,15}$

Síndrome compartimental: el síndrome compartimental es una complicación rara. En una revisión sistemática en el Children's Hospital of Los Angeles, se encontró una nula 
incidencia de esta complicación; ${ }^{15}$ sin embargo, en una publicación de la Revista Española de Cirugía Osteoarticular, Gómez Palacios la menciona como una complicación postoperatoria precoz.

Infección de las agujas de Kirschner: se han reportado tasas de hasta $1 \%$ de las infecciones superficiales y $0.2 \%$ de las infecciones profundas. Esto sugiere que un enfoque operativo seguro y la administración de antibióticos perioperatorios no está indicado. ${ }^{16}$

\section{Complicaciones tardías en el tratamiento}

Deformidad angular: Presencia de malunión (cúbito varo); suele ser una complicación común. ${ }^{17} \mathrm{La}$ incidencia de cúbito varo oscila desde 4 hasta $58 \%$, con una media de $30 \%$. El cúbito valgo, por el contrario, es más infrecuente, con una incidencia de 0 a $8.6 \%$.

Esta deformidad se evalúa midiendo el ángulo de acarreo del codo y se compara con el lado contralateral. Ésta es secundaria a una reducción inadecuada que conduce a malunión. El cúbito valgo y varo pueden causar tanto limitaciones funcionales como deformidades cosméticas. ${ }^{17}$

Pérdida de movilidad: la rigidez del codo es una complicación rara. Después de seis semanas de la fijación con agujas de Kirschner, es raro encontrar una diferencia en el rango de movimiento superior a 15 grados con el codo contralateral. La mayoría de los casos mejoran en unos pocos meses. ${ }^{17}$

Miositis osificante: es una complicación extremadamente rara. Se relaciona con fracturas que se han tratado de forma abierta y en las que se han realizado excesivas manipulaciones o han sido sometidas a fisioterapias muy agresivas.

Necrosis avascular de la tróclea: fue descrita por McDonnell y Wilson. Es una complicación rara tras una fractura supracondílea de húmero. La sintomatología aparece pasados los meses o los años, pero no compromete la función del codo.?
Otros: hay complicaciones que puede presentar el paciente pasadas unas semanas; es una nueva fractura sobre el codo que presentó anteriormente una fractura supracondílea de húmero.?

El objetivo del presente trabajo fue determinar la frecuencia de las complicaciones en fracturas supracondíleas humerales en menores de ocho años de nuestro hospital.

\section{Material y métodos}

Se realizó un estudio retrospectivo y observacional de cohorte. Se obtuvo la información de los expedientes clínicos de la población menor de ocho años que hubieran sido atendidos durante el período de Marzo de 2014 a Febrero de 2018, quienes además tuvieran diagnóstico de fractura supracondílea de húmero y a los cuales se les hubiera realizado un seguimiento en dicha institución. El criterio de inclusión fue: varones y mujeres menores a ocho años con fractura supracondílea de humero; el criterio de exclusión fue: trauma craneoencefálico, polifracturado; finalmente, se tomó como criterio de eliminación aquellos pacientes con enfermedades endocrinológicas.

A cada paciente, en su atención inicial en el Servicio de Urgencias, se le realizó una radiografía anteroposterior y lateral verdadera del codo afectado y otra del sano (comparativas). Los datos se tomaron a través del seguimiento en consulta externa, realizando una evaluación clínica y radiográfica a la una, dos, cinco semanas y tres, seis, 12 meses después del tratamiento definitivo.

Se utilizaron las siguientes variables cualitativas: niños, sexo masculino o femenino, solución de continuidad de la metáfisis distal del húmero por encima de la línea fisaria, valoración de la fuerza muscular, valoración de la sensibilidad, presencia o ausencia de pulsos y pacientes atendidos en este hospital. Las variables cuantitativas fueron: menores de ocho años, ángulo de Baumann con valor normal a 20 grados, ángulo de acarreo con una media de 15 grados en

Tabla 1: Frecuencia de complicaciones encontradas en fracturas supracondíleas.

Complicaciones de fracturas supracondíleas humerales en niños

\begin{tabular}{|c|c|c|c|c|c|}
\hline & & \multicolumn{4}{|c|}{ Complicaciones posteriores al tratamiento } \\
\hline \multicolumn{2}{|c|}{$\begin{array}{l}\text { Complicaciones previas } \\
\text { al tratamiento }\end{array}$} & \multicolumn{2}{|c|}{$\begin{array}{l}\text { Complicaciones precoces, en los } \\
\text { primeros días tras el tratamiento }\end{array}$} & \multicolumn{2}{|c|}{$\begin{array}{l}\text { Complicaciones tardías } \\
\text { en el tratamiento }\end{array}$} \\
\hline $\begin{array}{l}\text { Complicaciones } \\
\text { neurológicas }\end{array}$ & $1.44 \%(4)$ & Pérdida de reducción & $0 \%$ & Deformidad angular & $\begin{array}{c}3.97 \% \text { (11) } \\
\text { cúbito varo } \\
0.72 \%(2) \\
\text { cúbito valgo }\end{array}$ \\
\hline Complicaciones vasculares & $0 \%$ & Complicaciones neurológicas & $1.44 \%(4)$ & Pérdida de movilidad & $0.36 \%(1)$ \\
\hline \multirow[t]{3}{*}{ Síndrome compartimental } & $0 \%$ & Complicaciones vasculares & $0 \%$ & Miositis osificante & Rara \\
\hline & & Síndrome compartimental & Rara & $\begin{array}{l}\text { Necrosis avascular de la } \\
\text { tróclea }\end{array}$ & Rara \\
\hline & & $\begin{array}{l}\text { Infección de las agujas de } \\
\text { Kirschner }\end{array}$ & $0.72 \%(2)$ & Otros & Rara \\
\hline
\end{tabular}




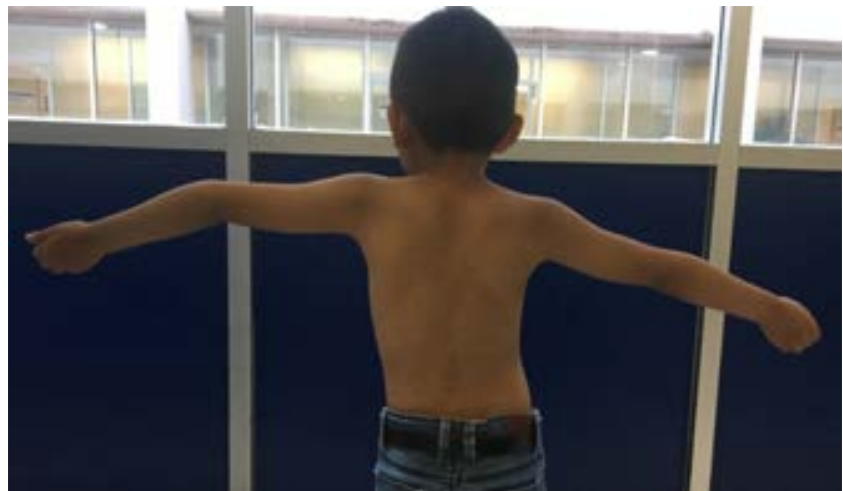

Figura 1: Deformidad angular cúbito varo.

mujeres y una media de 11 grados en varones y arcos de movilidad de la articulación del codo. Por último, se empleó, como escala nominal, la división de las complicaciones en precoces y tardías.

Para el análisis estadístico, se calcularon frecuencias simples, medidas de tendencia central según correspondió a cada variable. Todos los pacientes contaron con consentimiento informado, firmado por alguno de los tutores. No se contó con apoyo económico para este estudio.

\section{Resultados}

Se obtuvieron 277 pacientes en total. La edad de los pacientes fue variable: para el menor era de un año y el mayor de ocho años. De éstos, 59.9\% (166) de los pacientes era del sexo masculino y 40.07\% (111) era pacientes del sexo femenino. En 46.5\% (129) de las fracturas se afectó el codo izquierdo y 53.4\% (148) el codo derecho. Las complicaciones secundarias a fractura supracondílea humeral fueron:

\section{A) Complicaciones previas al tratamiento}

Se presentaron complicaciones neurológicas en 1.44\% (4) pacientes.

\section{B) Complicaciones posteriores al tratamiento}

1. Precoces: se presentaron complicaciones neurológicas en $1.44 \%$ (4) pacientes e infección de agujas de Kirchner en $0.72 \%$ (2) pacientes.

2. Tardías: cúbito varo 3.97\% (11) pacientes, cúbito valgo en $0.72 \%$ (2) pacientes, pérdida de la movilidad en $0.36 \%$ (1) paciente (Tabla 1).

\section{Discusión}

La principal complicación encontrada en este estudio fue cúbito varo (Figura 1) con 3.97\%; esta cifra en la literatura se describe con una frecuencia de 4 a $58 \%$, por lo que nuestros hallazgos son similares a lo reportado por la literatura mundial. Por lo anterior, se recomienda buscar por parte del cirujano una reducción exacta durante el acto quirúrgico para disminuir el porcentaje de esta complicación.

La segunda complicación más frecuente fueron aquellas neurológicas previas al tratamiento (Figura 2). En general, se reportaron en $1.44 \%$, es decir, una frecuencia baja y acorde con la literatura, ya que ésta se reporta de 6 a 42\%; consideramos que esto puede deberse a que en el Área de Urgencias generalmente se tiende a realizar una discreta manipulación de la fractura, sobre todo las tipo II y III. No obstante, esta manera de abordar las fracturas es discutida, ya que algunos autores no recomiendan manipular las fracturas en el Área de Urgencias. Por nuestra parte, consideramos que puede ser un punto importante en la disminución de complicaciones neurológicas, pues al mismo tiempo que se consigue una reducción anatómica de manera inicial, se consigue disminuir el riesgo de complicaciones vasculares, así como el síndrome compartimental; esto debido a que no se encontraron ninguna de estas últimas complicaciones.

En tercer lugar, notamos las complicaciones neurológicas precoces en los primeros días posteriores al tratamiento quirúrgico, las cuales aparecieron en $1.44 \%$. Esta cantidad en la literatura se describe con una frecuencia de $3.5 \%$ con la colocación de agujas de Kirschner con técnica cruzada, y en nuestro reporte es baja, respecto a lo anteriormente dicho. Asimismo, éstas se relacionaron directamente con la colocación de agujas de Kirschner con técnica cruzada, ya que sólo las presentaron pacientes en los cuales se realizó esta fijación. Cabe señalar que el retiro de la aguja de Kirschner presentó mejoría clínica del paciente con recuperación de la neuropatía.

La cuarta complicación encontrada fueron las infecciones de la aguja de Kirschner, las cuales se reportaron en $0.72 \%$. Esta suma en la literatura se encuentra con una frecuencia de infecciones superficiales en $1 \%$ y profunda en $0.2 \%$. Nuestro hallazgo al respecto es bajo en relación con

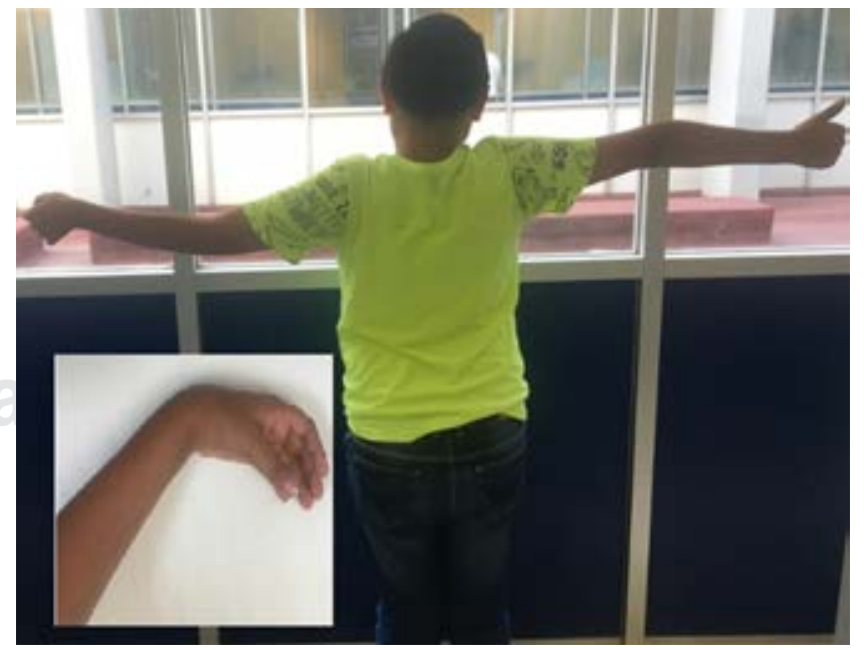

Figura 2: Lesión neurológica previa al tratamiento quirúrgico. En el recuadro, se observa la muñeca en flexión y los dedos con afectación secundaria a la lesión del nervio radial, sin embargo, en el seguimiento presentó una mejoría clínica progresiva. 
la literatura. El manejo transoperatorio es el siguiente: al ingresar a la sala, el cirujano realiza asepsia de extremidad torácica afectada con clorhexidina y, mediante un medioambiente quirúrgico estéril más aplicación de un antibiótico de manera profiláctica, se realiza el procedimiento quirúrgico. Con esto, suponemos que se disminuye el riesgo de infecciones. En este sentido, algunos autores recomiendan no utilizar antibiótico.

La quinta complicación encontrada es cúbito valgo. Ésta la encontramos en $0.72 \%$, cifra similar a la reportada en la literatura con $0-0.86 \%$. Por último, la pérdida de movilidad se presentó en un paciente, lo cual representa el 0.36\%; la literatura la reporta como una complicación rara, lo cual también coincide con nuestro reporte.

Con base en las complicaciones encontradas en los pacientes, es importante realizar una exploración física detallada en el Área de Urgencias, previamente al tratamiento definitivo y reportar si existe alguna lesión en ese momento. Esta fractura, que es muy común en menores de ocho años, es importante que reciba el manejo más adecuado en urgencias, así como su terapéutica temprana, a fin de disminuir en lo posible dichas complicaciones.

\section{Conclusiones}

La complicación más frecuente de las fracturas supracondíleas de húmero en este estudio fue el cúbito varo en pacientes menores de ocho años de edad. La segunda complicación fue la lesión neurológica previa al tratamiento quirúrgico.

Bibliografía

1. Rodríguez-Martínez JJ, Mora-Ríos FG, Mejía-Rohenes LC. Frecuencia de codo traumático pediátrico en el Hospital Regional General Ignacio Zaragoza. Rev Esp Med Quir. 2018; 23(2): 87-92.

2. Farrow L, Ablett AD, Mills L, Barker S. Early versus delayed surgery for paediatric supracondylar humeral fractures in the absence of vascular compromise. Bone Joint J. 2018; 100: 1535-41.

3. Barrón EA, Sánchez JF, Cruz JR. Perfil clínico-epidemiológico de las fracturas supracondíleas de húmero en pacientes pediátricos en un hospital general regional. Cirugía y Cirujanos. 2015; 83 (1): 29-34.
4. Corres IA, Torres A. Clasificación de las fracturas supracondíleas en niños: Gartland, AO y Holmberg. ¿Cuál tiene el mayor acuerdo interobservador? Acta Ortop Mex. 2015; 29(6): 299-302.

5. Vaquero A, González G, Moraleda L. Management of supracondylar fractures of the humerus in children. Efort Open Rev. 2018; 3 (10): 526-40.

6. Pimienta N, Castillo E, Matos JL, Aguila LR, Ballate D, Alfonso Y. Incidencia de las fracturas supracondíleas de codo en pediatría: fijación percutánea lateral. Acta Médica del Centro. 2018; 12(2): 140-6.

7. Gómez VE, Gil J, Herrera A. Complicaciones de las fracturas supracondíleas de húmero en la infancia. Revista Española de Cirugía Osteoarticular. 2013; 48: 150-62.

8. Barret K, Skaggs D, Sawyer J, Andras L, Moisan A, Goodbody C, Flynn J. Supracondylar humeral fractures with isolated anterior interosseous nerve injures: is urgent treatment necessary? J Bone Joint Surg Am. 2014; 96(1): 1793-7.

9. Kumar V, Singh A. Fracture supracondylar humerus: a review. J Clin Diagn Res. 2016; 10(12): 1-6.

10. Badkoobehi H, Choi P, Bae D, Skaggs D. Management of the pulseless pediatric supracondylar humeral fracture. J Bone Joint Surg Am. 2015; 97(11): 937-43.

11. Ladenhauf H, Schaffert M, Bauer J. The displaced supracondylar humerus fracture: indications for surgery and surgical options: a 2014 update. Curr Opin Pediatr. 2014; 26: 64-9.

12. Karim M, Hosny A, Mohamed N, Abdelatif N, Hegazy M, Awadallah $\mathrm{W}$, et al. Cossed wires versus 2 lateral wires in management of supracondylar fracture of humerus in children in the hans of junior trainees. J Orthop Trauma. 2016; 30: 123-8.

13. Khoshbin A, Leroux T, Wasserstein D, Wolfstadt J, Law P, Mahomed N, et al. The epidemiology of paediatric supracondylar fracture fixation: a population-based study. Injury Int J Care Injured. 2014; 45: 701-8.

14. Joiner E, Skaggs D, Arkader A, Andras L, Lightdale N, Pace Lee, Ryan D. Iatrogenic nerve injuries in the treatment of supracondylar humerus fractures: are we really just missing nerve injuries on preoperative examination? J Pediatr Orthop. 2014; 34: 388-92.

15. Gupta N, Kay R, Leitch K, Femin D, Tolo V, Skaggs D. Effect of surgical delay on perioperative complications and need for open reduction in supracondylar humerus fractures in children. $J$ Pediatr Orthop. 2004; 24: 245-8.

16. De Neira J, Prada A, Ciruelos R, Pretell J. Supracondylar humeral fractures in children: current concepts for management and prognosis. Int Orthop. 2015; 39(11): 2287-96. De SICOT Sitio web: https://www. ncbi.nlm.nih.gov/pubmed/26311512.

17. Dabis J, Daly K, Gelfer Y. Supracondylar fractures of the humerus in children-review of management and controversies. Orthop Muscular Syst. 2016; 5: 1-8.

Conflicto de intereses: El presente artículo no presenta conflicto de intereses. 